\title{
Nonlinear Friction and Resistance, Generating Sources of Optimal Points in the Energy Field of Agricultural Aggregates Working Process
}

\author{
Petru Cârdei \\ Computer engineering \\ INMA \\ Bucharest, Romania \\ petru_cardei@yahoo.com \\ Mircea Bădescu \\ Faculty of Mechanics \\ University of Craiova \\ Craiova, Romania \\ badescu.mircea@gmail.com \\ Nicolae Constantin \\ Soil Laboratory \\ INMA \\ Bucharest, Romania \\ nicu.constantin@yahoo.com
}

\author{
Alexandros Alexiou \\ Faculty of Mechanics \\ University of Craiova \\ Craiova, Romania \\ alexiou72@gmail.com
}

\author{
Valentin Vladut \\ Scientific Director \\ INMA \\ Bucharest, Romania \\ valentin_vladut@yahoo.com
}

\author{
Eugen Marin \\ Soil Laboratory
}

INMA

Bucharest, Romania

marin_43@yahoo.com

Received: March 2, 2019. Revised: April 13, 2021. Accepted: September 16, 2021. Published: November 15, 2021.

\begin{abstract}
The paper presents a point of view on the main sources that can generate some optimal points in the energy field of the agricultural machines working processes. It looks like a possible source of the existence of optimal points in the energetic field of work processes of agricultural machinery and equipment, are the coefficients of friction and specific resistance to deformation of soil. In the news models these coefficients became nonlinear functions. Similar forms are given for all three coefficients and is shown the existence of optimal points. They make some considerations about this method and include results obtained using it.
\end{abstract}

Keywords- Optimization, Energy Field, Nonlinear Frictions, Nonlinear Resistances

\section{INTRODUCTION}

The problem of optimal calculation of the energy of agriculture aggregates working process is a problem addressed since the first half of the twentieth century. The problem is even more in actuality, the more energy resources crisis has deepened and overlapped over the environmental crisis (environmental pollution caused by emissions generated by the fossil fuel consumption and mechanical pollution of the soil), and financial.

Saving energy and financial resources have become major objectives in the context of the demand for agricultural products increases, taking in consideration global population growth.

According to [6], the energy balance sheet is a balance sheet regarding to the sources and energy consumption, including losses in a system or a power plant. The power balance sheet, after [6] is an equilibrium relation developed to the motor shaft of a vehicle, tractor, etc.. and the amount of powers consumed for displacement, performing useful mechanical work and covering the losses.

Another important balance sheet is the traction balance sheet, that, after [6] is an equilibrium relation of the external forces which acting on the tractor or other traction vehicle, designed on the rolling track surface. The traction balance sheet allows the determination of the tractor working conditions for the various areas and use conditions, as shown in [5].

In [5], is also shows that, the traction balance sheet analysis, both practically and theoretically points of view have a great importance for optimization of the agricultural tractors exploitation. In [1] it is shown that, the energy consumption in agricultural works directly depends on the aggregates working capacity.

For this reason, the ability to work comes in optimization models of the agricultural aggregates working processes.

Besides, [1] is entirely dedicated to optimization problems of energy consumption, but this consumption cannot be 
optimized effectively, without taking into account a series of problems strictly related to it and the needs of agricultural exploitations: rational use of traction parameters of agricultural tractors, rational forming of agricultural aggregates, optimization of movement methods of agricultural aggregates, correlation of mechanization technologies with energy saving requirements, the introduction of automation in the agricultural aggregates driving, optimization of parameters regarding the agricultural machines and aggregates design.

Although, the problem of the agricultural aggregates energy optimization has a considerable age, solutions remained at the level of the optimal elementary calculus, whose foundations were laid in the first decades of the second half of the last century [1], [3], [4].

At this level, the introduction of some nonlinear components in the structure of the traction resistance forces is motivated only by getting some working speed functions, that allow obtaining optimal points in the strict sense (either by cancelling the first order derivatives, or by the method of Lagrange's multipliers).

From physical point of view, any interpretation of the new nonlinear coefficients introduced has not been given, in order to define the nonlinear terms (mostly second-degree terms in travel speed). Against this background, our research comes to fill this gap by introducing nonlinearities naturally, starting from empirical motivations.

\section{THE BASIC IDEA}

The proposed idea is the innovative one in relation to the literature studied. The point of view exposed starts from two sources. The first source is supplied by the bibliography, that address the part of aggregates energy optimization, [1], [3], [4], which introduces, in order to find some optimal points in the strict sense for the working process of the aggregates designed for soil tillage, dependencies of the head resistance of the second-degree terms in travel speed. The introduction of these terms is made in the friction forces with the ground or the composite ground with vegetal material (the composite material) (stubble). The second source is [2], that largely analyses the head resistance from theoretically and experimentally points of view and is one of the few sources that assert the presence of the second-degree terms in the formula for the head resistance calculation.

There are many generalizations in the literature dedicated to the phenomena of friction and resistance to deformation. These generalizations are common in the body's movement physics in the air, e.g., [8].

Our comments and solutions are related less to the first source, and are intimately related to the profound structure of the drag forces proposed in [2] and accepted in most literature in the field. Solidity of the assertions in [2] is based on experimental facts, that the author mentions them. However, in [2] slips a small inconsistency between the analytical structure of the drag formula (formula (24") :

$$
F_{t}=\left(\mu G_{m}+k a b\right)+\varepsilon \rho a b v^{2}
$$

and the graphical representation of Fig. 64 from the same source [2]. The author [2] mentions that, the "formula (24") shows that, by varying the speed and keeping other factors constant, the traction force required increases after a parabolic law. In formula (1) $F_{t}$ is the resistance force due to machine, $\mu$ is the coefficient of sliding friction of the machine to the ground, $G_{m}$ is the towed vehicle weight, $k$ is a coefficient characterizing specific resistance to deformation of the soil, $a$ and $b$ are the depth and the width of the towed farm machinery, $\varepsilon$ is a coefficient that depends on the active surface of the body working, $\rho$ is the soil bulk density and $v$ is the aggregate velocity. Traction force necessary to move the tractor's own is:

$F_{a}=f G_{t}$,

where $F_{a}$ is the force necessary for tractor move, $f$ is the friction coefficient tractor wheels running on the ground and $G_{t}$ is tractor weight.

Multiple determinations of the traction force made by Goreacikin also confirm in principle this situation, as evidenced by the data represented in Fig. 64, which shows the variation of the $F_{t}$ force according to speed, in case has ploughed at the same depth a field with $(k)$ specific resistance constant to different speeds. It is true that, this increase is quite low, indicating a low value of the last term of the relation (24"), compared with values of other terms.

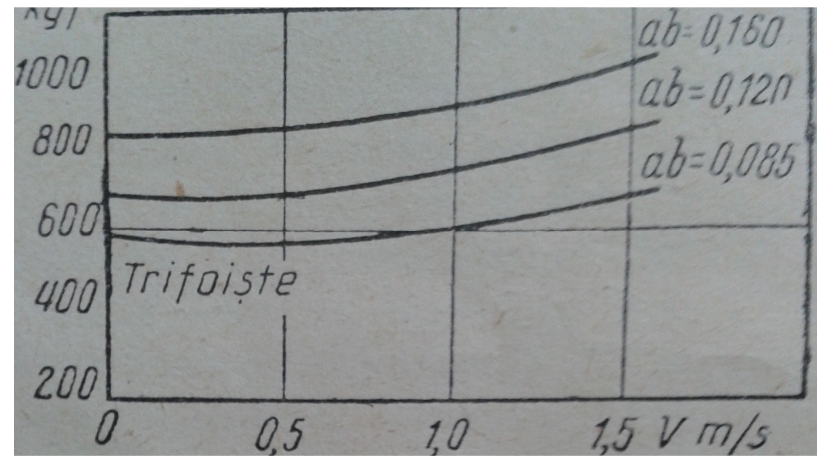

Fig. 1 Drag force dependence of speed, after [2].

The small inadvertence I have mentioned it between the formula (1), respectively (24") from [2] and the graph in Fig. 64 from the same [2], consists in that, some parabolas from the graphic representation have the point of minimum for a positive speed, while the formula (1) has the point of minimum exactly for the null value of the speed.

Therefore, the shape (1) of the drag force is susceptible to improvements, if the data in Fig. 1 represents the experimental data.

As inspiration source in order to improve the formula [2], it seems at least, an important solution, a suggestion that comes from the study of the friction forces from the source [7]. 

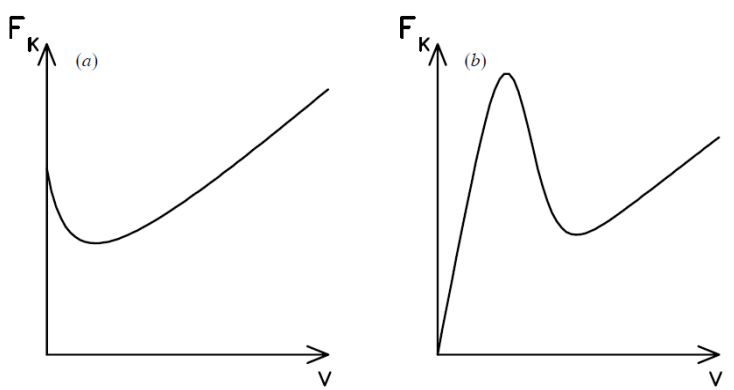

Fig. 2 Friction force - speed dependence, after [12].

Schematic shapes of the speed dependence of the friction forces occur in [7] and are presented by this paper in Fig. 2.

What will be new in this paper is the generalization of the dependence of working speed, both of the wheels rolling friction force on the ground (encouraged by [7]) and the resistance coefficient at soil deformation, which thus becomes a function of speed of the working body in the soil. Other sources in support of the proposed changes are: [9], [10], [11], [12], [13], [14]. Therefore, it proposes the following dependence formula for the friction coefficients:

$$
f(v)=f_{0}+f_{1} v+f_{2} v^{2}, \mu(v)=\mu_{0}+\mu_{1} v+\mu_{2} v^{2},
$$

respectively, for the resistance coefficient to the soil deformation:

$$
k(v)=k_{0}+k_{1} v+k_{2} v^{2},
$$

Coefficients of quadratic functions (3) and (4) relate to the important points on the charts and become:

$$
\begin{aligned}
& f(v)=f_{0}+2 \frac{f_{1}-f_{0}}{v_{f}} v+\frac{f_{0}-f_{1}}{v_{f}^{2}} v^{2}, \\
& \mu(v)=\mu_{0}+2 \frac{\mu_{1}-\mu_{0}}{v_{\mu}} v+\frac{\mu_{0}-\mu_{1}}{v_{\mu}^{2}} v^{2}, \\
& k(v)=k_{0}+2 \frac{k_{1}-k_{0}}{v_{k}} v+\frac{k_{0}-k_{1}}{v_{k}^{2}} v^{2}
\end{aligned}
$$

where $f_{0}, f_{l}, v_{f}, \mu_{0}, \mu_{l}, v_{\mu}, k_{0}, k_{l}, v_{k}$, are coefficients of friction and resistance functions.

If it is known experimental data, the newly introduced coefficients can be calculated using interpolation methods, e.g. the method of the least squares.

With the relations (5) the general shape of the drag force to traction (hook force) from a machine to seedbed preparation or soil tillage, generally becomes:

$$
F_{t}(v)=\mu(v) G_{m}+k(v) a b+\varepsilon \rho a b v^{2}
$$

or explaining the relations (5):

$$
\begin{aligned}
& F_{t}(v)=\mu_{0} G_{m}+k_{0} a b+ \\
& +2\left(\frac{\mu_{1}-\mu_{0}}{v_{\mu}} G_{m}+\frac{k_{1}-k_{0}}{v_{k}} a b\right) v+ \\
& +\left(\frac{\mu_{0}-\mu_{1}}{v_{\mu}^{2}} G_{m}+\frac{k_{0}-k_{1}}{v_{k}^{2}} a b+\varepsilon \rho a b\right) v^{2}
\end{aligned}
$$
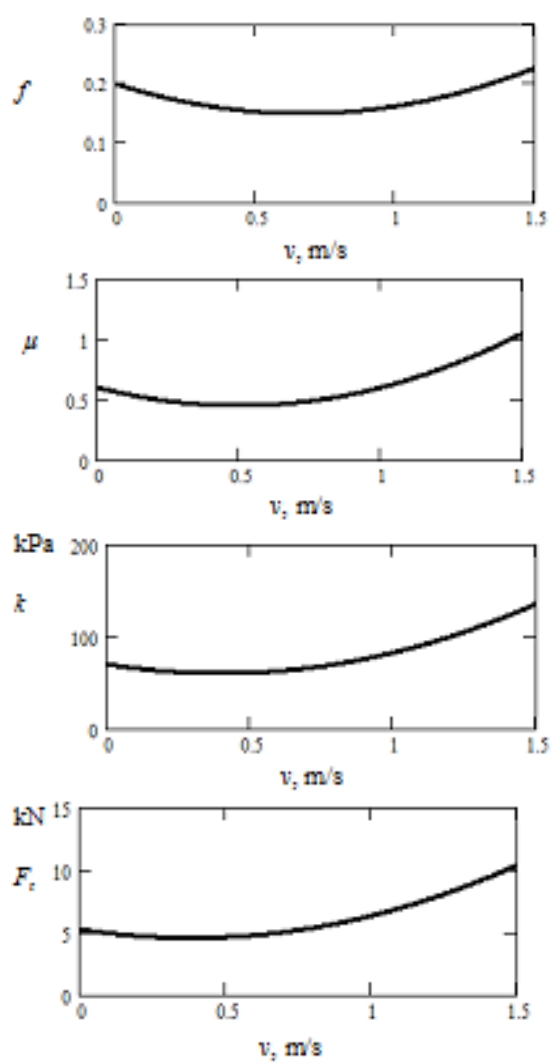

Fig. 3. The variations with speed of the friction coefficients, the resistance coefficient to deformation and the traction resistance force.

Similarly, adding to (7) the necessary force for the tractor self displacement, (2), it obtains the total force consumed by the tractor in order to displacement in work of the aggregate.

$$
\begin{aligned}
& F_{T}(v)=f_{0} G_{t}+\mu_{0} G_{m}+k_{0} a b+ \\
& +2\left(\frac{f_{1}-f_{0}}{v_{f}} G_{t}+\frac{\mu_{1}-\mu_{0}}{v_{\mu}} G_{m}+\frac{k_{1}-k_{0}}{v_{k}} a b\right) v+ \\
& +\left(\frac{f_{0}-f_{1}}{v_{f}^{2}} G_{t}+\frac{\mu_{0}-\mu_{1}}{v_{\mu}^{2}} G_{m}+\frac{k_{0}-k_{1}}{v_{k}^{2}} a b+\varepsilon \rho a b\right) v^{2}
\end{aligned}
$$

It is noted that, if $f_{l}=f_{0}=f, \mu_{l}=\mu_{0}=\mu, k_{l}=k_{0}=k$, we obtain the law (1). Also, if the speeds of the points of minimum of the three coefficients depending on the $v$ variable are very high, even if the equalities mentioned before do not occur, but these 
differences are negligible in comparison with the speeds of the points of minimum, the law (8) is reduced to (1).

One can advance the hypothesis that all or at least a part of the coefficients involved in defining the functions (3) - (5) may depend on other soil characteristics: moisture, chemical composition, content of gravel, sand, etc.

\section{MINIMIZING TOTAL FORCE NECESSARY WORKFLOW}

The total force needed is a component of aggregate dynamics intimately involved in energy unit. Starting from this force is calculated required power, then energy to the aggregate carrying workflow. Is found by simply differentiating with respect to speed, the force necessary to conduct the trial, (8), has a minimum point with coordinates:

$$
\begin{gathered}
v_{\min }=-\frac{\frac{f_{1}-f_{0}}{v_{f}} G_{t}+\frac{\mu_{1}-\mu_{0}}{v_{\mu}} G_{m}+\frac{k_{1}-k_{0}}{v_{k}} a b}{\frac{f_{0}-f_{1}}{v_{f}^{2}} G_{t}+\frac{\mu_{0}-\mu_{1}}{v_{\mu}^{2}} G_{m}+\frac{k_{0}-k_{1}}{v_{k}^{2}} a b+\varepsilon \rho a b}, \\
F_{T \text { min }}=\left[\left(\frac{f_{0}-f_{1}}{v_{f}^{2}} G_{t}+\frac{\mu_{0}-\mu_{1}}{v_{\mu}^{2}} G_{m}+\frac{k_{0}-k_{1}}{v_{k}^{2}} a b+\varepsilon \rho a b\right) .\right. \\
\left.\cdot\left(f_{0} G_{t}+\mu_{0} G_{m}+k_{0} a b\right)-\left(\frac{f_{1}-f_{0}}{v_{f}} G_{t}+\frac{\mu_{1}-\mu_{0}}{v_{\mu}} G_{m}+\frac{k_{1}-k_{0}}{v_{k}} a b\right)^{2}\right] . \\
\cdot\left(\frac{f_{0}-f_{1}}{v_{f}^{2}} G_{t}+\frac{\mu_{0}-\mu_{1}}{v_{\mu}^{2}} G_{m}+\frac{k_{0}-k_{1}}{v_{k}^{2}} a b+\varepsilon \rho a b\right)^{-1}
\end{gathered}
$$

The existence of the minimum point of the force is transferred power and energy. Optimal coordinates of the power and energy may differ from the force.

\section{MINIMIZING ENERGY}

Starting from total drag force, (8), and the aggregate velocity is calculated the required power for the working process. Power is integrated over time and obtains the energy needed to get the process. This calculation, simplified (consider piecewise constant speed, working process, $v$, turning speed, $v_{i}$, idle speed, $v_{g}$ ), is described in [16], [17], [18], [19] and [20]. In this way is obtains, for example, a complex tillage aggregate, the energy consumption following the next expression:

$$
\begin{aligned}
& E\left(v, v_{i}, v_{g}\right)=\left[f(v) G_{t}+k(v) a b+\mu(v) G_{m}+\varepsilon \rho a b v^{2}\right] . \\
& \cdot L\left(\left[\frac{C}{b}\right]+1\right)+f\left(v_{i}\right)\left(G_{t}+G_{m}\right) l_{i}\left(\left[\frac{C}{b}\right]+1\right)+f\left(v_{g}\right)\left(G_{t}+G_{m}\right) L_{s}
\end{aligned}
$$

where $L$ and $C$ are the plot length and width and $b$ is the operating width of the aggregate. In formula (10) the parameter $L_{s}$ is the length of the of path that runs for repairing the dislevelments. Thus, the energy consumed in the process becomes a function of three variables: $v, v_{i}$ and $v_{g}$. The functions $f, \mu$ and $k$ are given in (5). Then any energy extremum points (10), are found by solving the system of equations:

$$
\frac{\partial E}{\partial v}=0, \frac{\partial E}{\partial v_{i}}=0, \frac{\partial E}{\partial v_{g}}=0 .
$$

Performing derivatives, is obtains the system:

$G_{t} \frac{d f}{d v}+a b \frac{d k}{d v}+G_{m} \frac{d \mu}{d v}+2 \varepsilon \rho a b v=0$,

$\frac{d f}{d v_{i}}=0$

$\frac{d f}{d v_{g}}=0$.

with the solution:

$$
\begin{aligned}
& v_{\text {opt }}=\frac{G_{t} \frac{f_{0}-f_{1}}{v_{f}}+a b \frac{k_{0}-k_{1}}{v_{k}}+G_{m} \frac{\mu_{0}-\mu_{1}}{v_{\mu}}}{G_{t} \frac{f_{0}-f_{1}}{v_{f}^{2}}+a b \frac{k_{0}-k_{1}}{v_{k}^{2}}+G_{m} \frac{\mu_{0}-\mu_{1}}{v_{\mu}^{2}}+\varepsilon \rho a b}, \\
& v_{\text {iopt }}=v_{f}, \\
& v_{\text {gopt }}=v_{f} .
\end{aligned}
$$

(13) are the coordinates of the optimal point that minimize the energy. To obtain a measure of the energy performance independent of the surface worked, is can to work with the energy consumed per unit of area:

$$
E_{s}\left(v, v_{i}, v_{g}\right)=\frac{E\left(v, v_{i}, v_{g}\right)}{L b\left(\left[\frac{C}{b}\right]+1\right)} \text {. }
$$

Optimal energy point coordinates (14) are the same as the energy (13). Only the optimal energy value is changes.

For example, in the case of A 1800 tractor - BI-PLAN 700 combiner unit, characterized by the constants: $\delta=0.1193$ (tractor slip), $k_{0}=31500 \mathrm{~Pa}, k_{l}=30500, v_{k}=1.5 \mathrm{~m} / \mathrm{s}, \rho=1000$ $\mathrm{kg} / \mathrm{m}^{3}, f_{0}=0.2, f_{l}=0.17, v_{f}=1.78, \mu_{0}=0.5, \mu_{l}=0.4, v_{\mu}=1.7 \mathrm{~m} / \mathrm{s}$, $\varepsilon=2, m_{m}=3950 \mathrm{~kg}$ (roller), $m_{t}=10200 \mathrm{~kg}, \eta_{t r}=0.98, c=0.85$, $a=0.18 \mathrm{~m}, b=7.0 \mathrm{~m}, P_{e}=147.2 \mathrm{~kW}$, is obtains the next optimum coordinates points: $v=1.018 \mathrm{~m} / \mathrm{s}, v_{i}=1.78 \mathrm{~m} / \mathrm{s}, v_{g}=1.78 \mathrm{~m} / \mathrm{s}$. In these condition the productivity is $W=1.956$ ha per hour. The specific surface energy variation (14) around the optimal working point, depending on $v$ and the $v_{i}$, is represented in fig. 4 . 


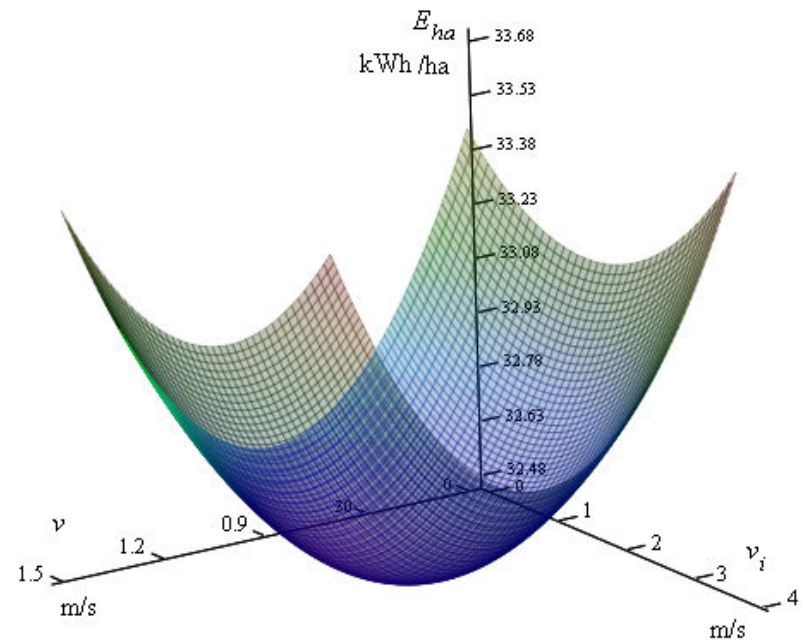

Fig. 4 The variation of the specific surface energy of the A1800-BI-PLAN 700 combiner aggregate, on $v$ and $v_{i}$ around the optimal point.

\section{CONCLUSIONS}

Research conducted in the obtaining of possible physical causes of optimal working points in the agricultural machinery business processes whose results are described in this article marks a border area.

Now the physical causes of the existence of optimal points in these work processes are at least partially substantiated. Other versions may differ only in the form of linear laws, not by physical fundamentals that define (i.e. coefficients of friction and resistance involved in the process).

The existence of optimal points is not ensured by the idea used. Nonlinear coefficients laws that define dependence variables involved in the nonlinear terms in speed, depending on the soil characteristics. One of the main soil characteristics involved in the process, is moisture. Depending on the soil characteristics, optimal working points may exist or not, or it is possible to transform the points of minimum in point of maximum. Optimal point's coordinates can be slightly modified depending on the physical characteristics of the soil. The connection between the nonlinear coefficients law and the soil physical characteristics remains a challenge at this level. We have no experimental data to formulate these laws and their formulation cannot be done without a considerable amount of experience.

Research developed based on the results presented in this article has allowed obtaining optimal working processes of agricultural aggregates. Energy domain of these processes is the space defined by energy consumption, fuel and working capacity. All results presented in this article, including the aforementioned consequences will be considered true only after experimental confirmation. Therefore the results presented in this article are only the beginning of a long and difficult road, which should clarify not only in agricultural aggregates optimize work processes, but also the physical relations between variables involved in this process.

\section{REFERENCES}

[1] A. Sandru, M. Badescu, L. Sandru, Reducing energy consumption through rational use of agricultural aggregates, Romanian Writing Publishing, Craiova, 1982.

[2] N. N. Letosnev, Agricultural Machinery, State Agro-Forestry Publishing House, Bucharest, 1959.

[3] C. Dobrescu, Optimization of parameters of the agricultural aggregates to reduce energy consumption, Editorial propaganda agricultural machinery, Bucharest, 1981.

[4] A. Sandru, S. Popescu, I. Cristea, V. Neculăiasa, Exploitation of agricultural machinery, Didactic and Pedagogic Publishing House, Bucharest, 1983.

[5] D. Toma, T. Neagu, I. Florescu, S. Lepşi, Agricultural Tractors, Didactic and Pedagogic Publishing House, Bucharest, 1978.

[6] Ş. Căproiu, V. Scripnic, M. Segărceanu, D. Toma \& all, Dictionary of agricultural mechanics, CERES Publishing House, Bucharest 1972.

[7] F., J. Elmer, Nonlinear dynamics of dry friction, J. Phys. A:Math. Gen. 30 (1997) 6057-6063.

[8] M. Rădoi, E. Deciu, Mechanics, . Didactic and Pedagogic Publishing House, Bucharest, 1981.

[9] M. Urbakh, J. Klafter, D. Gourdon and J. Israelachvili, The nonlinear nature of friction, Nature, vol. 430, 2004 , pp. 525-528.

[10] M. Vaishya and R. Singh, Sliding friction-induced non-linearity and parametric effects in gear dynamics, Journal of Sound and Vibration, 248(4), 2001, pp. 671-694.

[11] L.,R.Ray, Nonlinear Tire Force Estimation and Road Friction Identification: Simulation and Experiments, Automatica, vo. 33, issue 10, 1997, pp. 1819-1833.

[12] C. Makkar, Nonlinear modelling, identification, and compensation for frictional disturbances, A thesis presented to the graduate school of the University of Florida in partial fulfilment of the requirements for the degree of master of science, University of Florida, 2006.

[13] T., A. Shugar, Numerical modelling of steady-state plo/soil interaction, Technical Report TR-2081-SHR, Naval Facilitiues Engineering Service, Port Hueneme, California 93043-4370, 1997.

[14] W., R. Gill, Soil Dynamics in Tillage and Traction, Agricultural Research Service USDA, 1968.

[15] U. A. Rosa, D. Wulfsohn, Constitutive model for high speed tillage using narrow tools, Journal of Terramechanics 36, pp. 221-234, 1999.

[16] V. A. Meca, P. Cârdei, Studies and Researches for Unifying the Resistance Expression of machines Designed to Soil Tillage With Applications in the Working Regime Optimisation, INMATEH, vol. 36, pp. 21-26, 1/2012.

[17] P. Cârdei, A. Meca, G. Kostandinov, Soil farm machinery mode of operation:from the optimisation to the basis (1), INMATEH, vol. 37, pp.13-20, 2012.

[18] P. Cârdei, G. Kostandinov, Soil farm machinery mode of operation:from the optimisation to the basis (2), INMATEH, vol. 37, pp. 21-30, 2012;

[19] P. Cardei, R. Sfîru , I. Pirnă, M. Bădescu, S. Boruz, S. Lazar, Studies and researches on energetic optimisation of fodder harvesting combines-unidimensional models, INMATEH, vol. 38, pp. 5-14, 2012.

[20] P. Cardei, R. Sfîru , I. Pirnă, M. Bădescu, S. Boruz, At. Atanasov, Studies and researches on energetic optimisation of fodder harvesting combines-two idimensional models, INMATEH, vol. 38, pp. 15-22, 2012 .

\section{Creative Commons Attribution License 4.0 (Attribution 4.0 International, CC BY 4.0)}

This article is published under the terms of the Creative Commons Attribution License 4.0

https://creativecommons.org/licenses/by/4.0/deed.en_US 